\title{
New developments in transcatheter therapy of mitral valve disease
}

\author{
Claudia Walther ${ }^{1}$, Stephan Fichtlscherer ${ }^{1}$, Tomas Holubec ${ }^{2}$, Mariuca Vasa-Nicotera ${ }^{1}$, Mani Arsalan $^{2}$, \\ Thomas Walther ${ }^{2}$
}

${ }^{1}$ Department of Cardiology, ${ }^{2}$ Department of Cardiothoracic Surgery, The University of Frankfurt, Frankfurt, Germany

Contributions: (I) Conception and design: C Walther; (II) Administrative support: T Walther, S Fichtlscherer; (III) Provision of study materials or patients: M Arsalan; (IV) Collection and assembly of data: C Walther; (V) Data analysis and interpretation: M Arsalan; (VI) Manuscript writing: All authors; (VII) Final approval of manuscript: All authors.

Correspondence to: Claudia Walther, MD, PhD. Department of Cardiology, University Hospital Frankfurt, Theodor Stern Kai 7, 60590 Frankfurt, Germany. Email: claudia.walther@kgu.de.

\begin{abstract}
Mitral valve regurgitation (MR) belongs to one of the most common acquired valve diseases in western countries with increasing prevalence in older age. For patients with high perioperative risk and older age prohibitive for valve surgery, the development of transcatheter mitral valve therapies offers new options. Assessment of the severity and etiology of MR and thorough imaging of the mitral valve anatomy and pathology are necessary prerequisites for appropriate decision making in the field of transcatheter mitral valve therapies. Different transcatheter repair and replacement techniques are on the market, most of them mimicking surgical techniques. With some techniques (e.g., the MitraClip device), there is good clinical experience ( $>80,000$ devices implanted worldwide), and evidence (three randomized studies), whereas for newer procedures, safety and efficacy data are still very limited. Transcatheter mitral repair and replacement techniques have to be considered as complementary treatment options for high-risk patients indicated by the Heart Teams. The different techniques and devices will be introduced and discussed in the following paper.
\end{abstract}

Keywords: Mitral valve regurgitation (MR); functional mitral regurgitation; degenerative mitral regurgitation; percutaneous mitral repair; transcatheter mitral valve repair; heart failure; MitraClip device

Submitted Sep 05, 2019. Accepted for publication Dec 30, 2019.

doi: $10.21037 /$ jtd.2019.12.137

View this article at: http://dx.doi.org/10.21037/jtd.2019.12.137

\section{Background}

\section{Introduction}

Mitral valve (MV) disease is amongst the most common valvular heart defects worldwide, and the majority of patients suffer from mitral valve regurgitation. Its incidence is $1-2 \%$ of the western population, and it is more prevalent with age affecting up to $10 \%$ of the population above 75 years (1). The presence and the severity of mitral regurgitation have a prognostic impact on long-term survival (2-4). The mortality rate of untreated severe mitral regurgitation is up to $50 \%(5,6)$. However, approximately $50 \%$ of patients meeting the indication for mitral valve therapy are not referred to surgery $(7,8)$ due to heart failure, older age or other comorbidities (9). Therefore alternative, less invasive treatment strategies are required; some already are in routine clinical practice. In the following, we will discuss the different interventional approaches to treat MV disease.

When considering transcatheter therapies for MV disease, it is of great importance to understand the etiology of the mitral valve regurgitation (MR). MR is either designated to a "degenerative/primary" (related to abnormalities of the anatomical structures of the mitral valve like leaflets or chordae tendineae) or to a "functional/ secondary" etiology (based on systolic tethering of the anatomically intact leaflets due to ventricular dysfunction (ischemic or other) or atrial dilatation). Before treating 
MV regurgitation, a thorough evaluation of its etiology is necessary and optimal medical therapy of the underlying disease should be performed before any interventional therapy.

\section{Management of care}

The current ESC Guidelines on valvular heart disease emphasize the importance of a multidisciplinary heart team (including general and interventional cardiologists, cardiac surgeons, imaging specialists, HF specialists, and cardiac anesthesiologists) for evaluation and therapy planning in patients suitable for transcatheter mitral valve therapy (10).

In primary $\mathrm{MR}$, medical therapy may be a temporary option to improve clinical symptoms but is not a lasting and curative therapeutic approach. Surgical treatment is the treatment of choice to correct severe primary MR in symptomatic patients (10). Mitral valve repair is the preferred method compared to mitral valve replacement considering lower perioperative mortality, superior long term survival, and reduced valve related complications (11).

In secondary MR optimization of medical therapy is recommended (10) before considering interventional therapies. Cardiac resynchronization therapy may lead to a reduction in secondary MR in selected patients with a wide QRS complex (12). According to the current guidelines only weak recommendations are given for surgery (level of recommendation IIa) and transcatheter mitral valve therapy (level of recommendation IIb) in this group of patients with secondary MR (10).

\section{Anatomical and imaging considerations}

The mitral valve is a complex anatomical structure consisting of the annulus, two leaflets (anterior and posterior leaflet, which are both arranged of three segments) and the chordae tendineae, which connect the leaflets to the left ventricle. Thorough imaging evaluation and analysis has to be performed to allocate the best suitable therapy. Transthoracic (TTE) and transesophageal echocardiography (TEE) are essential investigations to comprehensively evaluate the mitral valve apparatus and to get an idea about the pathology and anatomical structures involved in MV disease. The echocardiographic preand periprocedural assessment of the mitral valve should be performed following certain standard views $(13,14)$. The application of real-time three-dimensional 3D TEE can provide better visualization of the mitral valve and its pathology. In addition to echocardiographic imaging CT scanning of the mitral apparatus can give additional information about calcifications and geographical relations of $M V$ apparatus and adjacent structures.

\section{Transcatheter mitral valve therapy}

Over the past years an increasing number of transcatheter MV therapies was developed and there is further technical progress. The available devices are based on very variable techniques and different modes of actions and may be applied according to the underlying MV pathology. Those new developments may be generally categorized into procedures targeting:

(I) Leaflet repair;

(II) Direct or indirect annuloplasty;

(III) Implantation of artificial chordae;

(IV) Transcatheter mitral valve replacement.

Table 1 gives an overview of the most commonly applied MV repair techniques.

\section{Leaflet repair/edge-to-edge technique}

\section{MitraClip}

The MitraClip (Abbott Laboratories, Menlo Park, California, USA) device consists of cobalt chromium and is covered with a polypropylene tissue (Figure 1). The MitraClip device has two arms and is based on the principle of approximating the edges of the anterior and posterior MV leaflet segments. This technique was designed after the surgical "edge-to-edge" repair technique, first introduced by Alfieri et al. (15). The MitraClip device received CEMark approval in Europe in 2008 and FDA approval in the USA in 2013 for use in primary MR and very recently 2019 for use in secondary MR. Currently the third generation of the MitraClip (offering two sizes: NTR in original size and XTR with $3 \mathrm{~mm}$ longer clip arms) is on the market with improved steering, navigational and positioning clip capabilities to facilitate precise positioning and accurate results (Figure 2).

To achieve a preferably high success rate considering MR reduction certain anatomical requirements of the MV should be considered. The following MR criteria were identified to be favorable anatomical parameters for a successful Clip implantation: leaflet coaptation length $>2 \mathrm{~mm}$, coaptation depth $<11 \mathrm{~mm}$, in case of primary MR-flail gap < $10 \mathrm{~mm}$ and flail width $<15 \mathrm{~mm}$. With growing experience regarding the MitraClip implantation also more complex 
Table 1 Overview of transcatheter mitral valve repair devices which have already CE approval and devices with promising techniques

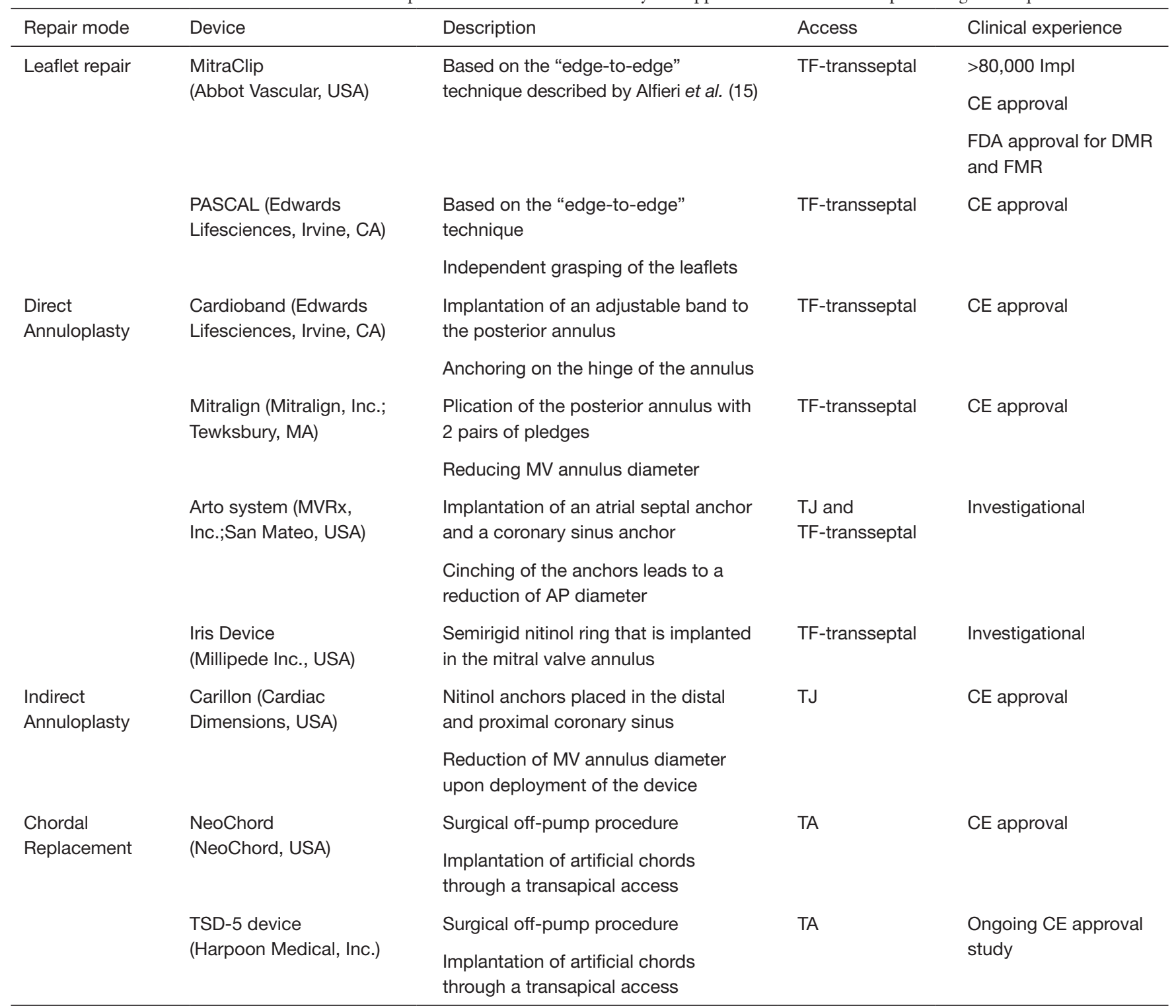

TF, transfemoral; TJ, transjugular; TA, transapical; MV, mitral valve; AP, anterior-posterior.

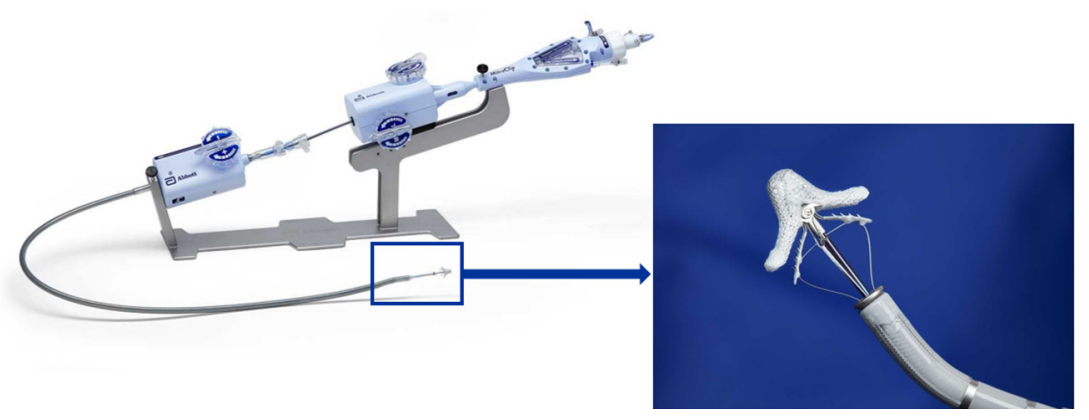

Figure 1 The MitraClip system with steerable console outside the body and the amplified MitraClip (with copyright permission from Abbot). 

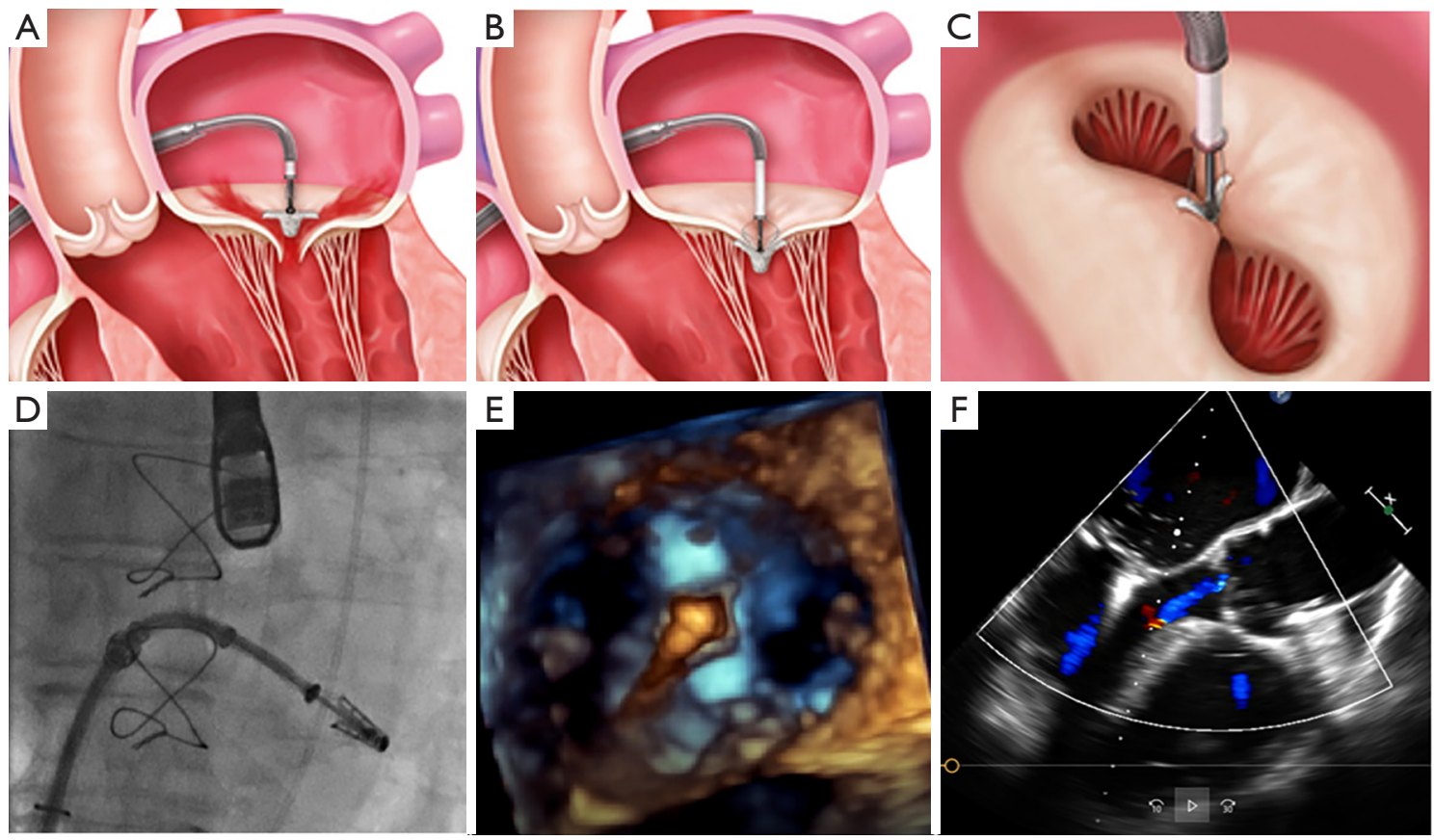

Figure 2 Standard steps of the MitraClip procedure. (A) Perpendicular positioning of the MitraClip above the mitral valve. (B) Grasping of the leaflets and closing of the MitraClip. (C) The typical "double orifice" after MitraClip implantation. (D) Fluoroscopic control of the correct position of the MitraClip. (E) 3D echocardiographic control of stable perpendicular position after advancing the MitraClip in the left ventricle. (F) The echocardiographic result after successful MitraClip implantation [figure with copyright permission from (16)].

valve morphologies and pathologies are treatable with the MitraClip device.

\section{Procedure technique}

The MitraClip procedure is usually performed under general anesthesia in most of the implanting centers. The intervention is guided using TEE and fluoroscopy in the catheterization laboratory. The MitraClip device is inserted using a transfemoral venous access a $24 \mathrm{~F}$ guiding catheter. Optimal transseptal puncture is a very important precondition for a successful procedure. In case of primary MR and large prolapse a more posterior and higher puncture site ( $5 \mathrm{~cm}$ above the annular plane) is recommended whereas in secondary MR puncture height should be around 3.5 to $4 \mathrm{~cm}$ above the annular plane, since tethering of the leaflets results in a coaptation level below the annular plane. The $24 \mathrm{~F}$ guiding catheter is then advanced in the left atrium over a stiff wire. After the Clip has been positioned over the MV it will be aligned perpendicular to the MV coaptation line using TEE guidance. Subsequently the MitraClip will be advanced into the left ventricle and after confirming the correct position in the MV pathology the leaflets will be captured and the Clip can be closed. The last maneuvers will be performed under continuously TEE control using standard views (intercommissural view and LVOT view). The MitraClip can be reopened and repositioned if necessary and in case of large degenerative MV defects or in secondary MR with large coaptation defects the implantation of two or more Clips may be necessary to achieve the desired results. Before releasing the Clip it is very important to assess the transmitral gradient to avoid functional mitral stenosis. The residual mean transmitral gradient should not exceed $5 \mathrm{mmHg}$. Patients tolerate a residual MR better than an increased mean transmitral gradient $>5 \mathrm{mmHg}$ (17). In case of complicated grasping application of adenosine or rapid pacing may facilitate leaflet capture. A common sequelae of the MitraClip procedure is the iatrogenic ASD which is usually tolerated very well. Relevant shunting over the iatrogenic ASD with increased right heart stress may have a negative prognostic impact and should be corrected (18).

Complications after the MitraClip procedure comprise bleeding, pericardial effusion, partial leaflet detachment, device embolization, endocarditis, mitral stenosis requiring 
surgery, left ventricular assist device implantation, devicerelated complications and are reported to occur in $3.2 \%$ to $13 \%$ of the procedures.

\section{Clinical evidence}

The MitraClip system is the most commonly used transcatheter repair technique with most interventions being performed in Europe so far. The clinical evidence for this device is derived from three randomized clinical studies:

In the EVEREST II study (19), 279 patients with severe $M R$ and indication for surgery were randomized in a 2:1 ratio to percutaneous mitral valve repair with the MitraClip device $(\mathrm{n}=184)$ or to surgical mitral valve repair or replacement therapy $(n=95)$. In most patients $(73 \%)$ the etiology of MR was of primary origin with very defined and restricted echocardiographic inclusion criteria. The combined primary efficacy endpoint after 12 months (freedom from death, freedom from surgery for MV dysfunction, and freedom from grade 3 and $4 \mathrm{MR}$ ) was reached in $55 \%$ of patients in the MitraClip group and $73 \%$ in the surgery group. The main driver for this significant difference was the high rate of surgery due to $M V$ dysfunction after MitraClip therapy ( $20 \%$ vs. $2.2 \%$ in the surgery group). However the safety endpoint was in favor of the MitraClip system mainly driven by a higher rate of bleeding requiring a blood transfusion in surgical patients. In this trial no device embolization did occur, and no mitral stenosis was reported and very interestingly surgical $\mathrm{MV}$ reconstruction was still possible after having a MitraClip implanted (20). The safety results of this trial led to a high acceptance of this percutaneous device worldwide.

Five-year results of the EVEREST trial demonstrated no significant difference in mortality rates between the two treatment groups (20.8\% in the MitraClip group and 26.8\% in the surgical repair group, $\mathrm{P}=0.4)$. And similar to the 12 months results the combined endpoint (freedom of death, MV surgery and grade 3 to $4 \mathrm{MR}$ ) was significantly lower in the MitraClip group (44.2\%) compared to the surgical group $(64.3 \%)(\mathrm{P}=0.01)$. The difference in the composite endpoint was mainly due to increased rates of grade 3 and $4 \mathrm{MR}$ and surgery in the percutaneous repair group. $78 \%$ of the surgeries in the percutaneous repair group occurred during the first six months of the study. After six months rates of grades 3 and $4 \mathrm{MR}$ and rates of surgery were comparable in both groups, which is a strong indicator for the long-term durability of the MitraClip device. As already mentioned, patients enrolled in the EVEREST trial were eligible for surgical therapy and therefore highly selected. But also results of observational studies on the commercial use of the MitraClip device in mainly primary MR confirmed a high procedural success rate of $>90 \%$, a mortality rate $<3 \%$ and overall 30-day serious complication rate was described to be $10-15 \%(21,22)$. In real-world registries like the German TRAMI registry (23) or the Italian GRASP registry (24) a good safety profile with a high success rate and good clinical results could be observed, even in patients with a higher risk profile and mainly secondary MR etiology. The experience with the MitraClip device is continuously growing giving the opportunity to expand the indication to a broader patient cohort with more complex MV anatomy and pathology.

Very recently two randomized controlled trials compared MitraClip to medical therapy in patients with secondary MR due to $L V$ dysfunction $(25,26)$ :

* The COAPT trial is an industry-sponsored prospective randomized trial, performed in the USA, comparing MitraClip therapy versus guideline-directed medical therapy (GDMT) in 614 patients with severe secondary MR and LV. The clinical endpoints were significantly improved in the MitraClip group compared to the control group showing a reduced hospitalization rate due to heart failure after 24 months (35.8\%) compared to the medical therapy group (67.9\%). In addition the all-cause mortality rate was lower in the MitraClip group $(29.1 \%)$ compared to medical therapy alone (46.1\%). MitraClip related complications after 12 months were recorded in $3.4 \%$ of patients.

* The MITRA-FR trial is an investigator-initiated prospective randomized trial and was performed in several centers in France. 304 symptomatic patients with relevant MR and LV dysfunction were randomly assigned to either percutaneous mitral valve repair (MitraClip) ( $\mathrm{n}=152)$ or medical therapy group $(\mathrm{n}=152)$. After 12 months there was no difference between both groups regarding mortality rate $(24.3 \%$ in the MitraClip group $v s$. $22.4 \%$ in the medical group) and hospitalizations due to the worsening of heart failure $(48.7 \%$ in the MitraClip group $v s .47 .4 \%$ in the medical group). Device related complication rate 12 months after MitraClip therapy was $14.6 \%$.

Possible explanations for these discrepant results are differences in study design and patients' characteristics. Patients enrolled in the MITRA FR trial had a more disproportional MR with more enlarged LV diameters. Furthermore the periprocedural success rate was better 

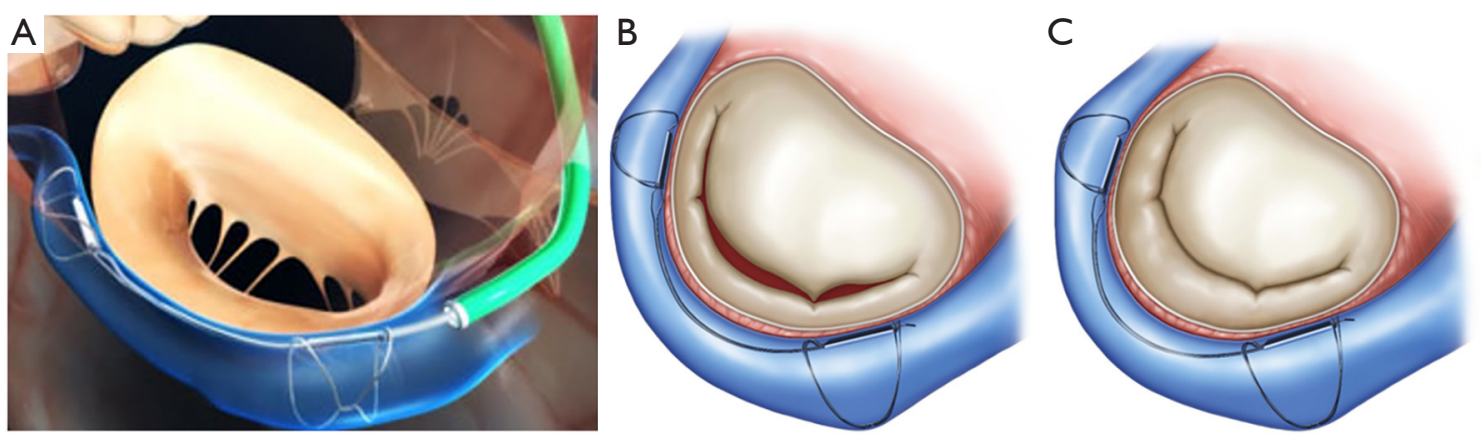

Figure 3 Scetch of the Carillon device procedure. (A) Positioning of the Carillon device with the two anchors in the great cardiac vein and the ostium of the coronary sinus. Deployed Carillon device before (B) and after "cinching" (C) with the restoration of leaflet coaptation [figure with copyright permission from (16)].

in the COAPT trial and patients in the control group in the COAPT trial were more refractory to medical therapy therefore less prone to improvement during the study follow up. Finally there was a longer follow up (24 months) in the COAPT trial, which was very important since differences in the clinical endpoints emerged beyond the 12 months follow up.

Although most of the etiologies of MR (primary and secondary) can be treated with the MitraClip device there is a considerable number of patients with severe MR who do not meet the anatomical criteria for Clip implantation or who have contraindications for Clip therapy. Several alternative devices have emerged on the market of percutaneous mitral valve therapy to treat MR.

\section{PASCAL transcatheter mitral valve repair (TMVr) system}

The PASCAL transcatheter device (Edwards Lifesciences, Irvine, CA) received CE-approval in Germany in 2019 and is similar to the MitraClip system-based on the edgeto-edge repair technique. The device was designed to overcome some of the MitraClip's technical limitations: broader "paddles" with a central spacer should facilitate $M R$ reduction and are thought to reduce stress and tethering on the valve leaflets, independent movement of the "paddles" should enable a better leaflet grasping especially in complex anatomies, and device elongation should facilitate maneuvers in the left ventricle. Procedural steps resemble those of the MitraClip therapy with access via the right femoral vein (27), using the transseptal approach and advancing the PASCAL implant over a $22 \mathrm{~F}$ guide catheter in the left ventricle. Clinical evidence for the PASCAL system is still limited. In the first observational study in 23 patients the PASCAL system was implanted by compassionate use (28). Residual $M R \leq 2$ was observed in $96 \%$ of the patients, no increased transmitral gradient could be detected despite the large implant device. Direct procedure related complications occurred in 2 patients (9\%) due to bleeding and transient ischemic attack. In the CLASP study (29) the safety and efficacy of the PASCAL system were evaluated in 62 patients with grades 3 to $4 \mathrm{MR}$. After 30 days, MAE was $6.5 \%$ with an overall mortality rate of $1.6 \%$. Residual MR $\leq 2+$ could be observed in $98 \%$ of these patients after 30 days with a corresponding functional improvement. These promising results have to be confirmed in a larger patient cohort and with long term follow up. A direct head-to-head comparison with the well-established MitraClip device will be performed in the ongoing CLASP II trial.

\section{Indirect annuloplasty devices}

\section{Carillon Mitral Contour System ${ }^{\mathrm{TM}}$}

The Carillon Mitral Contour System ${ }^{\mathrm{TM}}$ (Cardiac Dimensions, USA) is a right heart transcatheter indirect annuloplasty system for the treatment of patients with functional MR. The implantable device consists of a proximal and a distal self-expanding nitinol anchor connected via a shaping ribbon (Figure 3). The Carillon System is applied through trans-jugular venous access over a 9F delivery catheter and introduced into the coronary sinus. Utilizing the proximity of the coronary sinus to the mitral annulus apparatus, the mitral annulus diameter will be reduced during deployment of the device and consequently functional MR will be reduced (30). During the intervention imaging of the left circumflex artery (LCX) is recommended 

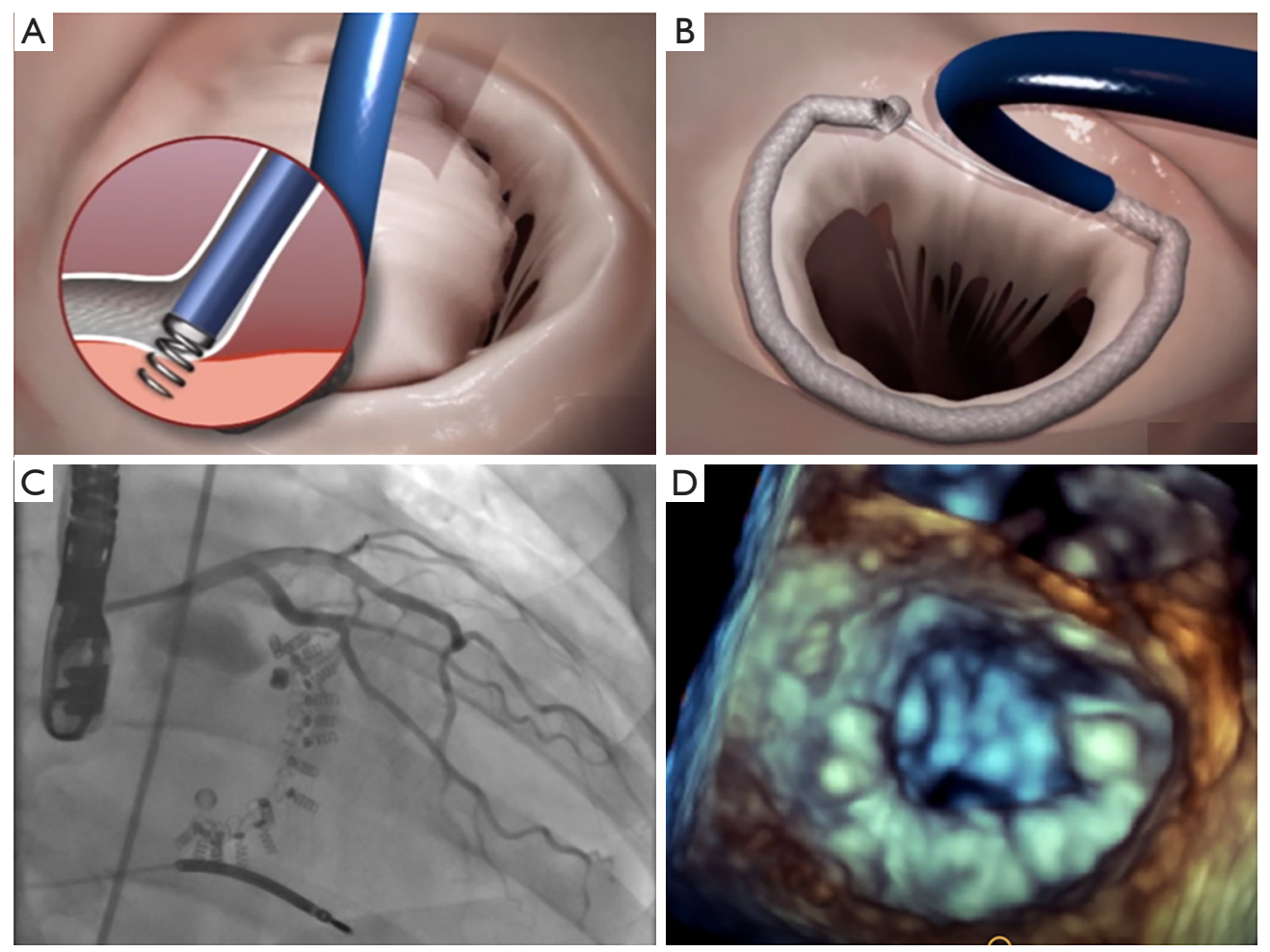

Figure 4 Procedural steps of the Cardioband system. (A) The Cardioband is anchored to the hinge of the posterior mitral annulus starting on the anterolateral commissure and reaching the posteromedial commissure. After deployment of the Cardioband (B) cinching of the Cardioband is performed to reduce annular diameters and enhance leaflet coaptation. (C) Fluoroscopic and angiographic control is performed to confirm the correct position of the device and to exclude any damage of the left circumflex artery. (D) 3D echocardiographic imaging ("en face view") after deployment of the Cardioband.

to exclude compression of the LCX which is located close to the mitral annulus. The advantages of the Carillon system are its simple use, benign design and the possibility of recapturing and repositioning the system if needed. The initial studies evaluating the CARILLON system showed an improvement of clinical symptoms, a reduction of MR and an improvement of $\mathrm{LV}$ remodeling parameters (20). Due to its design and its specific localization the CARILLON device was exposed to high strain at the level of the proximal anchor and asymptomatic device fractures in this area were observed in $25 \%$ of the cases. Therefore a modified device was developed and evaluated in the prospective single arm safety TITAN II trial (31). The improved clinical and echocardiographic results from the previous studies could be confirmed in the TITAN II trial. There was only one single device fracture $(2.8 \%)$ and the primary endpoint (30 days MAE) was $2.8 \% .12$ months mortality rate was $23 \%$ and none of the deaths was device-related (31). Meanwhile the Carillon System has been implanted in the $1000^{\text {th }}$ patient in
July 2019 demonstrating its acceptance in the percutaneous mitral valve repair area. A very clever designed double-blind randomized trial (REDUCE-FMR trial) was performed to evaluate the efficacy of the Carillon system objectively and preliminary results (presented at the TCT conference 2018, still unpublished) showed a reduction in MR and LV remodeling parameters.

\section{Direct annuloplasty devices}

\section{Cardioband system}

The Cardioband device (Edwards Lifesciences, Irvine, CA) is a percutaneous direct annuloplasty device (Figure 4) for the treatment of functional MR. It is designed according to surgical annuloplasty devices but applied via a percutaneous transseptal approach. Cardioband is an adjustable posterior band implanted from the anterolateral to the posteromedial commissure by multiple small anchors at the hinge of the posterior annulus. The implantation procedure is 


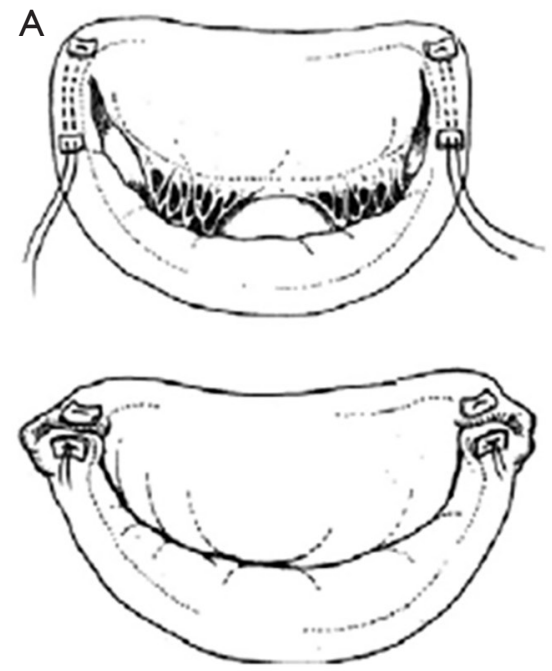

B

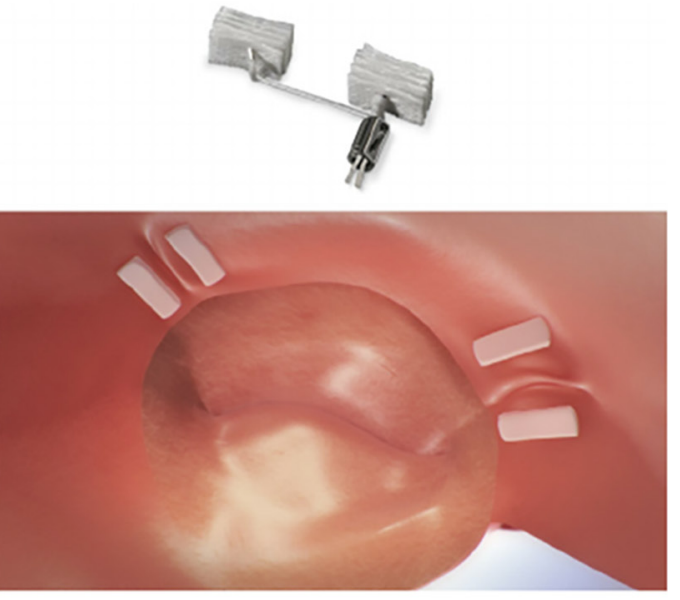

Figure 5 The Mitralign device mimicking a modified Kay Wooler annuloplasty. (A) Schematic imaging of the Mitralign device. (B) Plication of the posterior annulus with pledges.

performed under continuous echocardiographic (TEE) and fluoroscopic guidance. Good imaging quality is a condition sine qua no in this procedure. After the fixation of the band on the posterior annulus, the implant is adjusted under imaging control to reduce mitral annulus diameters, enhance leaflet coaptation and therefore reduce MR (32).

In a multicenter study of 60 patients treated with the Cardioband device the one year results demonstrated reasonable performance and safety results: One year survival rate was $87 \%$, moderate or less MR was present in $61 \%$ and functional status, quality of life and exercise capacity improved significantly. The Cardioband device had some technical problems in the early phase of the study. 9 of 10 patients in the study had an anchor disengagement leading to an inefficient device in five patients. As a consequence the device was modified in the first half of the study. Two coronary artery occlusions were reported during the study. After the improvement of screening no more coronary occlusions had been observed.

\section{Mitralign system}

The Mitralign annuloplasty system (Mitralign, Inc.; Tewksbury, MA) is predicated on the plication of the posterior annulus using a pledget delivery system via retrograde aortic access to the left ventricle and left atrium (Figure 5). Pairs of pledgets are placed at the opposite ends of the annulus to reduce annulus diameter resulting in reduced MR. The Mitralign system received CE Mark approval in 2016 due to the safety and efficacy endpoints reported in the CE Mark trial. Data from the prospective, multi-center, single arm study demonstrated a significant improved 6-minute walk test and improvement in left ventricular dimensions and remodeling after 6 months. Reduction of MR was achieved in $50 \%$ of the patients and device success was $70.4 \%$, pericardial tamponade occurred in $8.9 \%$, there was no death (33).

\section{Chordal replacement}

\section{The NeoChord DS1000 device}

The NeoChord device (NeoChord, Inc.) is a surgical off-pump mitral valve repair procedure for patients with degenerative MR. The procedure is performed under general anesthesia using a transapical access device and implanting artificial chords into the mitral valve leaflets. After the fixation of the Neochordae in the defined leaflet area, the chordae will be externalized and anchored at the ventricular access site. The results of the FIH TACT trial (34) led to CE Mark approval of the device in 2012. All of the 30 patients enrolled in the TACT trial had a prolapse of the PML with chordal rupture or elongation. Six patients needed reoperation due to failed repair. Since 2012, the NeoChord device was applied in $>450$ patients and there is an ongoing registry. In addition an FDA pivotal study (RECHORD trial) has been started aiming to enroll 585 patients at 20 participating centers.

\section{TSD-5 mitral valve repair device}

The TSD-5 mitral valve repair device (Harpoon Medical, 
Inc.) is a similar device compared to the above mentioned NeoChord device with transapical access. The artificial cords are implanted under TEE and fluoroscopic guidance at the posterior mitral leaflet with a special delivery system. After reassuring the optimal length of the chordae they will be fixed outside the left ventricle with Teflon material. The first results of the device in 13 patients were reported at the Transcatheter Cardiovascular Therapeutics Conference in 2016 showing a $100 \%$ procedural success rate and two reoperations due to pericardial effusions and two reoperations due to recurrent MR in the midterm follow up (35). There is an ongoing CE Mark study at 22 centers in five EU countries.

\section{Transcatheter MV replacement (TMVR)}

The complex anatomy of the mitral valve (D-shaped, asymmetric annulus, complex subvalvular apparatus, and large and variable dimensions) poses big handicaps in the field of TMVR. The main challenges of developing optimal TMVR prostheses are anchoring the prostheses in a D-shaped, noncalcified native valve preventing migration of the prosthesis into the left ventricle and avoiding a paravalvular leak and LVOT obstruction by displacing the anterior mitral leaflet into the LVOT. Considerable valve designs have been created to address all these challenges, some of them already under investigational use in patients (35).

\section{Access site}

For TMVI two access options exist: Transfemoral venous with transseptal access to the left atrium and the MV and transapical access to the left ventricle and the MV.

Transfemoral venous access supposedly is least invasive and after transseptal puncture offers an antegrade approach to the MV. Anatomically, however, any application system for a transcatheter mitral valve (TMV) has to be tilted to reach the native MV. Coaxial alignment of a valve cannot be accomplished before unsheathing in all instances. Therefore a transfemoral transseptal approach to the MV can be somewhat challenging.

Transapical access requires anterolateral minithoracotomy. This is slightly more invasive than a transfemoral puncture, however, it is a routine minimally invasive procedure and with a soft tissue retractor a non-rib-spreading approach can be used. Through an apical purse-string, a straight approach to the MV is obtained. This allows for precise positioning, anchoring, and implantation of any transcatheter MV prosthesis.

\section{Prostheses}

Different valve prostheses using different approaches, different anchoring, and sealing mechanisms are available. The overall worldwide experience with different devices is still limited. All valves consist of a self-expanding frame (usually Nitinol) that incorporates xenogenic leaflets to obtain a trileaflet biological valve. The most common tissue material is bovine or porcine pericardium, and porcine valve leaflets are an alternative. Table $S 1$ gives an overview of the most commonly used TMVI prostheses.

\section{Anatomy/functional morphology of MV}

In order to safely implant any TMVI prosthesis, several anatomical factors, which may be influencing the functional morphology of the heart, need to be considered. The MV itself is a very complex structure, and it is embedded quite closely to the left ventricular myocardium, papillary muscles, native mitral valve chordae and leaflets, and the left ventricular outflow tract (LVOT).

Any LVOT obstruction needs to be avoided. Therefore the mitral aortic angle should be $>120^{\circ}$, and severe septal hypertrophy should be excluded. Furthermore, any TMVI prosthesis should be placed as atrial as possible.

In order to avoid such complications, exact preoperative screening using precise transesophageal echocardiographic and CT assessment should be performed. Several factors need to be considered:

(I) Sufficient annular diameter for safe anchoring and sealing. The larger the annular diameter, however, the larger may be the risk for LVOT-obstruction.

(II) Engagement of the native leaflets for precise positioning and good sealing of a device.

(III) Consideration of the functional anatomy of the LVOT in order to avoid any obstruction after valve implantation.

(IV) Assessment of left ventricular function, ejection fraction may decrease after valve implantation with a sudden decrease in mitral incompetence.

\section{Clinical evidence}

The overall number of TMVI procedures is still limited worldwide. At present, according to data presented at EuroPCR Conference 2019 , slightly more than 500 patients have 
received TMVI worldwide. Amongst them, approximately $50 \%$ received a Tendyne prosthesis, approximately $25 \%$ an Intrepid prosthesis, approximately $12.5 \%$ a Tiara prosthesis, $3.5 \%$ a Caisson prosthesis, $3 \%$ a CardiaQ prosthesis, $3 \%$ an M3/Sapien prosthesis and 3\% a Highlife prosthesis.

In a recent literature overview of published studies on TMVR, it was concluded that TMVR is a feasible, less invasive alternative for treating severe mitral regurgitation in patients with high or prohibitive surgical risk. TMVR was associated with a high rate of successful valve implantation and excellent hemodynamic results. However, peri-procedural complications and all-cause mortality were relatively high (36). In that review, 308 patients who had received a TMVR were included. Mean patient age was $75.2 \pm 3.5$ years, mean STS score was $7.7 \pm 0.75$, and secondary; functional mitral incompetence was present in $77.5 \%$ of the patients. All-cause 30 -day mortality was $13.6 \%$, the stroke rate was $2.9 \%$, moderate or severe mitral incompetence was present in $1.5 \%$, and unplanned mitral valve surgery had to be performed in $4 \%$ of the patients. At a midterm follow-up of 10.1 months, mortality was $27.6 \%$, moderate or severe mitral valve incompetence was not diagnosed in any of 83 examined patients, unplanned mitral valve re-intervention rate was $2.3 \%$, device embolization occurred in $0.8 \%$, and endocarditis was seen in $2.7 \%$ of the patients, respectively.

When evaluating those preliminary data, we have to keep in mind that patient inclusion into any of the existing clinical trials is quite selective. A French study group examined factors associated with inclusion or rejection for TMVR trials between November 2016 and July 2018. Amongst 40 screened patients, $60 \%$ were refused. They concluded that despite good clinical profiles, patients could not be treated with TMVR because of mismatch between their anatomy and prosthesis characteristics (37).

\section{Conclusions}

Transcatheter mitral valve therapies are still in the early phase of their development-with the exception of the MitraClip device-with limited clinical evidence but promising results regarding safety and efficacy. Transcatheter mitral valve therapies are rapidly developing, and further technical refinements of the devices, together with increased experiences and improved learning curves of the operators, will allow for increased applications in the future.

To achieve these goals, it is very important to collect further data and to evaluate the existing transcatheter devices against standard care. The development of imaging techniques with a combination of fluoroscopic, echocardiographic, and computed tomographic data will further improve the technical success rate of procedures and reduce the complication rate to achieve the best possible result for the patient.

A very important aspect in this exciting field of new and fast-growing technologies is the existence of a Heart Team that builds the fundament for decision making and delivering the best quality of care to our patients through inter- and multidisciplinary cooperation.

\section{Acknowledgments}

Funding: None.

\section{Footnote}

Provenance and Peer Review: This article was commissioned by the Guest Editor (Ion S. Jovin) for the series "Interventional Cardiology" published in Fournal of Thoracic Disease. The article was sent for external peer review organized by the Guest Editor and the editorial office.

Conflicts of Interest: All authors have completed the ICMJE uniform disclosure form (available at http://dx.doi. org/10.21037/jtd.2019.12.137). The series "Interventional Cardiology" was commissioned by the editorial office without any funding or sponsorship. CW received honoraria from Abbot Vascular. TW received honoraria from Abbot Vascular and Edwards Lifesciences. SF received honoraria from Abbot Vascular and Edwards Lifesciences. Mariuca Vasa-Nicotera received honoraria from Abbot Vascular and Edwards Lifesciences. TH and MA have no other conflicts of interest.

Ethical Statement: The authors are accountable for all aspects of the work in ensuring that questions related to the accuracy or integrity of any part of the work are appropriately investigated and resolved.

Open Access Statement: This is an Open Access article distributed in accordance with the Creative Commons Attribution-NonCommercial-NoDerivs 4.0 International License (CC BY-NC-ND 4.0), which permits the noncommercial replication and distribution of the article with the strict proviso that no changes or edits are made and the 
original work is properly cited (including links to both the formal publication through the relevant DOI and the license). See: https://creativecommons.org/licenses/by-nc-nd/4.0/.

\section{References}

1. Nkomo VT, Gardin JM, Skelton TN, et al. Burden of valvular heart diseases: a population-based study. Lancet 2006;368:1005-11.

2. Grigioni F, Enriquez-Sarano M, Zehr KJ, et al. Ischemic mitral regurgitation: long-term outcome and prognostic implications with quantitative Doppler assessment. Circulation 2001;103:1759-64.

3. Koelling TM, Aaronson KD, Cody RJ, et al. Prognostic significance of mitral regurgitation and tricuspid regurgitation in patients with left ventricular systolic dysfunction. Am Heart J 2002;144:524-9.

4. Enriquez-Sarano M, Avierinos JF, Messika-Zeitoun $\mathrm{D}$, et al. Quantitative determinants of the outcome of asymptomatic mitral regurgitation. $\mathrm{N}$ Engl J Med 2005;352:875-83.

5. Mirabel M, Iung B, Baron G, et al. What are the characteristics of patients with severe, symptomatic, mitral regurgitation who are denied surgery? Eur Heart J 2007;28:1358-65.

6. Goel SS, Bajaj N, Aggarwal B, et al. Prevalence and outcomes of unoperated patients with severe symptomatic mitral regurgitation and heart failure: comprehensive analysis to determine the potential role of MitraClip for this unmet need. J Am Coll Cardiol 2014;63:185-6.

7. Vesely MR, Benitez RM, Robinson SW, et al. Surgical and Transcatheter Mitral Valve Repair for Severe Chronic Mitral Regurgitation: A Review of Clinical Indications and Patient Assessment. J Am Heart Assoc 2015;4. doi: 10.1161/JAHA.115.002424.

8. Grasso C, Capodanno D, Tamburino C, et al. Current Status and Clinical Development of Transcatheter Approaches for Severe Mitral Regurgitation. Circ J 2015;79:1164-71.

9. Iung B, Baron $\mathrm{G}$, Butchart EG, et al. A prospective survey of patients with valvular heart disease in Europe: The Euro Heart Survey on Valvular Heart Disease. Eur Heart J 2003;24:1231-43.

10. Baumgartner H, Falk V, Bax JJ, et al; ESC Scientific Document Group. 2017 ESC/EACTS Guidelines for the management of valvular heart disease. Eur Heart J 2017;38:2739-91.

11. Lazam S, Vanoverschelde JL, Tribouilloy C, et al. Twenty-
Year Outcome After Mitral Repair Versus Replacement for Severe Degenerative Mitral Regurgitation: Analysis of a Large, Prospective, Multicenter, International Registry. Circulation 2017;135:410-22.

12. van Bommel RJ, Marsan NA, Delgado V, et al. Cardiac resynchronization therapy as a therapeutic option in patients with moderate-severe functional mitral regurgitation and high operative risk. Circulation 2011;124:912-9.

13. Wunderlich NC, Siegel RJ. Peri-interventional echo assessment for the MitraClip procedure. Eur Heart J Cardiovasc Imaging 2013;14:935-49.

14. Guarracino F, Baldassarri R, Ferro B, et al. Transesophageal Echocardiography During MitraClip ${ }^{\circledR}$ Procedure. Anesth Analg 2014;118:1188-96.

15. Alfieri O, Maisano F, De Bonis M, et al. The doubleorifice technique in mitral valve repair: A simple solution for complex problems. J Thorac Cardiovasc Surg 2001;122:674-81.

16. Walther C, Fischer-Rasokat U, Nef H. Interventionelle Mitralklappen-Therapien. Klinikarzt 2017;46:146-152.

17. Neuss M, Schau T, Isotani A, et al. Elevated Mitral Valve Pressure Gradient After MitraClip Implantation Deteriorates Long-Term Outcome in Patients With Severe Mitral Regurgitation and Severe Heart Failure. JACC Cardiovasc Interv 2017;10:931-9.

18. Schueler R, Ozturk C, Wedekind JA, et al. Persistence of iatrogenic atrial septal defect after interventional mitral valve repair with the MitraClip system: a note of caution. JACC Cardiovasc Interv 2015;8:450-9.

19. Feldman T, Foster E, Glower DD, et al. Percutaneous repair or surgery for mitral regurgitation. $\mathrm{N}$ Engl J Med 2011;364:1395-406.

20. Argenziano M, Skipper E, Heimansohn D, et al. Surgical revision after percutaneous mitral repair with the MitraClip device. Ann Thorac Surg 2010;89:72-80; discussion $\mathrm{p} 80$.

21. Sorajja P, Mack M, Vemulapalli S, et al. Initial Experience With Commercial Transcatheter Mitral Valve Repair in the United States. J Am Coll Cardiol 2016;67:1129-40.

22. Nickenig G, Estevez-Loureiro R, Franzen O, et al. Percutaneous mitral valve edge-to-edge repair: in-hospital results and 1-year follow-up of 628 patients of the 20112012 Pilot European Sentinel Registry. J Am Coll Cardiol 2014;64:875-84.

23. Schillinger W, Hunlich M, Baldus S, et al. Acute outcomes after MitraClip therapy in highly aged patients: results from the German TRAnscatheter Mitral valve 
Interventions (TRAMI) Registry. EuroIntervention 2013;9:84-90.

24. Attizzani GF, Ohno Y, Capodanno D, et al. Extended use of percutaneous edge-to-edge mitral valve repair beyond EVEREST (Endovascular Valve Edge-toEdge Repair) criteria: 30-day and 12-month clinical and echocardiographic outcomes from the GRASP (Getting Reduction of Mitral Insufficiency by Percutaneous Clip Implantation) registry. JACC Cardiovasc Interv 2015;8:74-82.

25. Stone GW, Lindenfeld J, Abraham WT, et al. Transcatheter Mitral-Valve Repair in Patients with Heart Failure. N Engl J Med 2018;379:2307-18.

26. Obadia JF, Messika-Zeitoun D, Leurent G, et al. Percutaneous Repair or Medical Treatment for Secondary Mitral Regurgitation. N Engl J Med 2018;379:2297-306.

27. Grasso C, Popolo Rubbio A. The PASCAL transcatheter mitral valve repair system for the treatment of mitral regurgitation: another piece to the puzzle of edge-to-edge technique. J Thorac Dis 2017;9:4856-9.

28. Praz F, Spargias K, Chrissoheris M, et al. Compassionate use of the PASCAL transcatheter mitral valve repair system for patients with severe mitral regurgitation: a multicentre, prospective, observational, first-in-man study. Lancet 2017;390:773-80.

29. Lim DS, Kar S, Spargias K, et al. Transcatheter Valve Repair for Patients With Mitral Regurgitation: 30-Day Results of the CLASP Study. JACC Cardiovasc Interv 2019;12:1369-78.

30. Siminiak T, Wu JC, Haude M, et al. Treatment of functional mitral regurgitation by percutaneous

Cite this article as: Walther C, Fichtlscherer S, Holubec T, Vasa-Nicotera M, Arsalan M, Walther T. New developments in transcatheter therapy of mitral valve disease. J Thorac Dis 2020;12(4):1728-1739. doi: 10.21037/jtd.2019.12.137 annuloplasty: results of the TITAN Trial. Eur J Heart Fail 2012;14:931-8.

31. Lipiecki J, Siminiak T, Sievert H, et al. Coronary sinusbased percutaneous annuloplasty as treatment for functional mitral regurgitation: the TITAN II trial. Open Heart 2016;3:e000411.

32. Messika-Zeitoun D, Nickenig G, Latib A, et al. Transcatheter mitral valve repair for functional mitral regurgitation using the Cardioband system: 1 year outcomes. Eur Heart J 2019;40:466-72.

33. Nickenig G, Schueler R, Dager A, et al. Treatment of Chronic Functional Mitral Valve Regurgitation With a Percutaneous Annuloplasty System. J Am Coll Cardiol 2016;67:2927-36.

34. Seeburger J, Rinaldi M, Nielsen SL, et al. Off-pump transapical implantation of artificial neo-chordae to correct mitral regurgitation: the TACT Trial (Transapical Artificial Chordae Tendinae) proof of concept. J Am Coll Cardiol 2014;63:914-9.

35. Gammie JS, Bartus K, Gackowski A, et al. BeatingHeart Mitral Valve Repair Using a Novel ePTFE Cordal Implantation Device: A Prospective Trial. J Am Coll Cardiol 2018;71:25-36.

36. Del Val D, Ferreira-Neto AN, Wintzer-Wehekind J, et al. Early Experience With Transcatheter Mitral Valve Replacement: A Systematic Review. J Am Heart Assoc 2019;8:e013332.

37. Coisne A, Pontana F, Tchetche D, et al. Transcatheter Mitral Valve Replacement: Factors Associated with Screening Success and Failure. EuroIntervention 2019;15:e983-9. 
Table S1 Overview of transcatheter mitral valve replacement prostheses with a promising perspective

\begin{tabular}{|c|c|c|c|}
\hline Valve & Access & Specific features & Images \\
\hline $\begin{array}{l}\text { Tendyne (Abbott } \\
\text { Vascular, Abbott } \\
\text { Park, IL) }\end{array}$ & $\begin{array}{l}\text { TA, TF under } \\
\text { develop-ment }\end{array}$ & $\begin{array}{l}\text { - Nitinol stent, porcine pericardial leaflets } \\
\text { - Apical tether for fixation } \\
\text { - } \text { Fully repositionable and retrievable } \\
\text { - } \text { Largest clinical experience worldwide } \\
\text { - MAC substudy } \\
\text { - } \text { CE approval expected }\end{array}$ & \\
\hline $\begin{array}{l}\text { Intrepid (Medtronic, } \\
\text { Minneapolis, MN) }\end{array}$ & TA & $\begin{array}{l}\text { - Conformable outer stent to engage MV } \\
\text { annulus and leaflets } \\
\text { - Flexible brim to aid imaging } \\
\text { - Inner } 27 \mathrm{~mm} \text { stent with tri-leaflet bovine } \\
\text { pericardial valve }\end{array}$ & \\
\hline Tiara (Neovasc Inc.) & TA & $\begin{array}{l}\text { D-shaped valve, bovine pericardial leaflets, } \\
\text { Nitinol frame } \\
\text { - Three anchors }\end{array}$ & \\
\hline $\begin{array}{l}\text { CardiaQ (Edwards } \\
\text { Lifesciences Corp., } \\
\text { Irvine) }\end{array}$ & TF transseptal & $\begin{array}{l}\text { - Nitinol stent with relatively open frame } \\
\text { - Bovine pericardial leaflets } \\
\text { - Atrial and ventricular anchors } \\
\text { - Supraannular position to avoid LVOT } \\
\text { obstruction } \\
\text { - Intraannular sealing skirt } \\
\text { - TA approach not pursued }\end{array}$ & - \\
\hline $\begin{array}{l}\text { Caisson (LivaNova } \\
\text { PLC, UK) }\end{array}$ & TF transseptal & $\begin{array}{l}\text { - Composite system with anchor to the MV } \\
\text { and then valve placement inside the anchor }\end{array}$ & \\
\hline $\begin{array}{l}\text { Cardiovalve } \\
\text { (Cardiovalve) }\end{array}$ & TF transseptal & $\begin{array}{l}\text { - Self-expanding valve } \\
\text { - Short profile for minimal protusion into } \\
\text { the LVOT } \\
\text { - Specific anchoring and sealing meachanism } \\
\text { - Successful FIM } 2018\end{array}$ & \\
\hline $\begin{array}{l}\text { M3/Sapien } \\
\text { (Edwards } \\
\text { Lifesciences Corp., } \\
\text { Irvine) }\end{array}$ & TF transseptal & $\begin{array}{l}\text { Composite system: } \\
\text { - Anchoring dock that corrals native } \\
\text { - MV leaflets } \\
\text { - Sapien M3 valve is then inserted into the } \\
\text { anchoring dock }\end{array}$ & \\
\hline $\begin{array}{l}\text { Highlife (Highlife } \\
\text { SAS, Paris) }\end{array}$ & $\begin{array}{l}\text { TA/TF transseptal } \\
\text { and TF retrograde } \\
\text { arterial }\end{array}$ & $\begin{array}{l}\text { Composite system: } \\
\text { - Ring placed at MV annulus via TF } \\
\text { retro-grade approach } \\
\text { - Transcatheter prosthesis placed TA or TF } \\
\text { transseptally inside the ring }\end{array}$ & \\
\hline
\end{tabular}

TF, transfemoral; TA, transapical; MAC, mitral annular calcification; MV, mitral valve; LVOT, left ventricular outflow tract; FIM, first in man. 